\title{
Improved melting of latent heat storage via porous medium and
}

\section{uniform Joule heat generation}

\author{
Hayder I. Mohammed ${ }^{1}$, Pouyan Talebizadehsardari, ${ }^{2,}$, Jasim M. Mahdi $^{3}$, Adeel Arshad ${ }^{2}$, \\ Adriano Sciacovelli ${ }^{4}$, Donald Giddings ${ }^{2}$ \\ ${ }^{1}$ Department of Physics, College of Education, University of Garmian, Kurdistan, Iraq. \\ ${ }^{2}$ Fluids \& Thermal Engineering (FLUTE) Research Group, Faculty of Engineering, University of \\ Nottingham, Nottingham NG7 2RD, United Kingdom \\ ${ }^{3}$ Department of Energy Engineering, University of Baghdad, Baghdad 10071, Iraq. \\ ${ }^{4}$ Birmingham Centre for Energy Storage, School of Chemical Engineering, University of \\ Birmingham, UK
}

\section{Abstract}

To enhance the rate of heat transfer in phase change materials (PCM), high conductivity porous materials have been widely used recently as a promising method. This study introduces a novel approach for improving melting of PCM by incorporating uniform Joule heat generation with the porous structure compared to central heat generation. Different cases based on the heaterin foam configuration under the same heat generation rate are numerically verified and compared with the case of using the central heating element, which the heat transfer in the domain enhances by the porous medium. The effects of pore density and rate of heat generation are explored using the thermal non-equilibrium model to better deal with the interstitial heat transfer between the internal heat-generated-in-foam and the PCM. For the case with the central heating element, the effects of heater dimensions as well as the rate of heat generation are also investigated. The results show that the uniform heat generation from the porous structure can

\footnotetext{
${ }^{*}$ Corresponding author.

E-mail address: pouyan.talebizadehsardari@nottingham.ac.uk (P. Talebizadehsardari).
} 
24 substantially reduce the melting time. Applying $100 \mathrm{~kW} / \mathrm{m}^{3}$ for the rate of heat generation reduces the melting time by $21 \%$ compared with the best case of the localised heater. Meanwhile, applying higher pore-density foam does not bring any significant effect due to the uniform distribution of the heat generation. The results also show a small effect of localized heater size on the melting time with the same rate of heat generation density from the porous structure. However, for an identical volumetric heat source power of the localised heater, the rate of heat generation per volume is more effective compared with the heating element size due to the presence of the porous medium.

Keywords: Internal heat generation; Joule heating; Latent heat storage; Porous medium; Central heating element; Thermal non-equilibrium model.

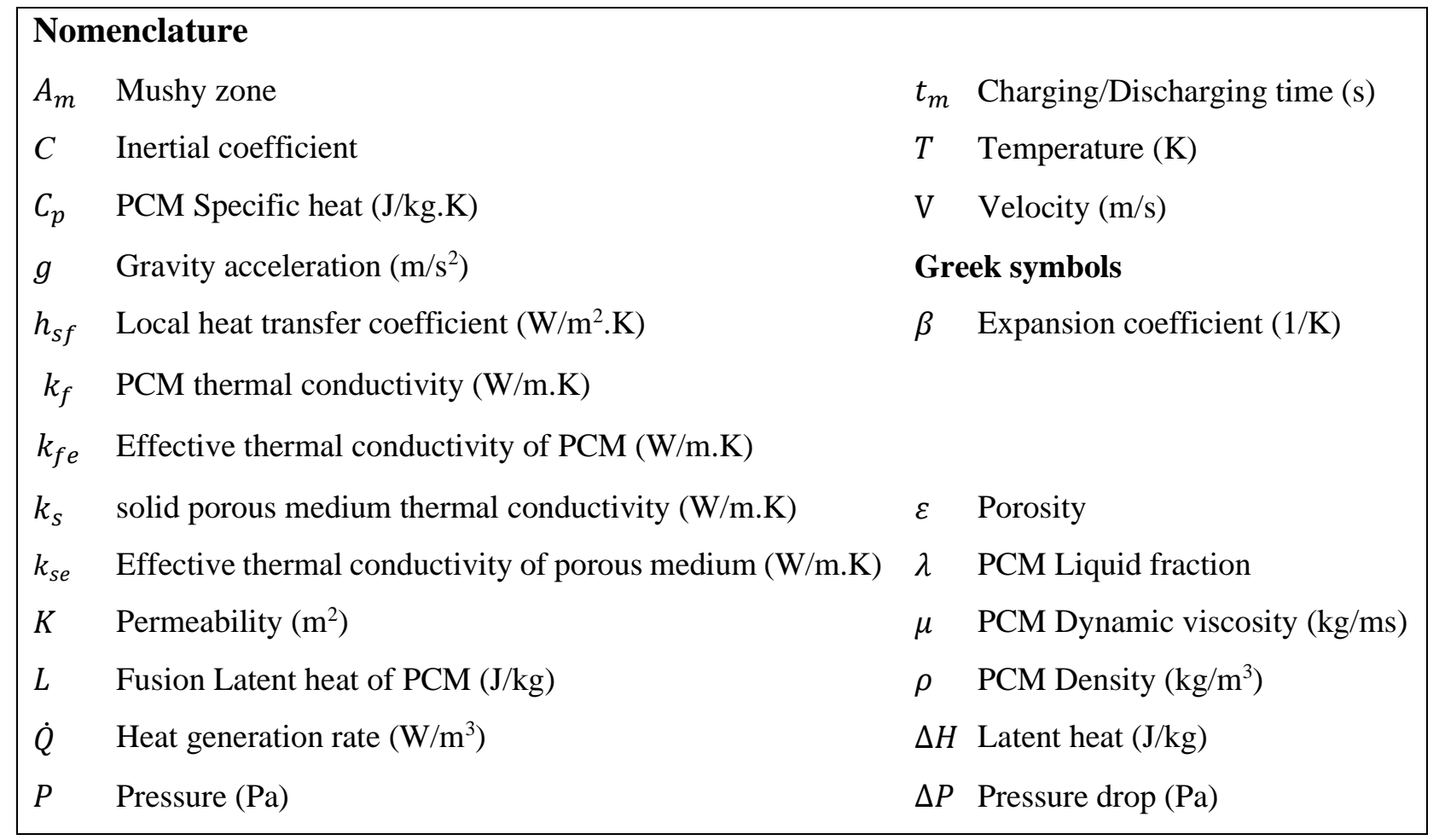

\section{Introduction}

Globally, fossil fuel still provides about $80 \%$ of the world's energy demand [1]. However, there are considerable release of greenhouse gases and pollutants (i.e.: $\mathrm{CO}, \mathrm{CO}_{2}$ and $\mathrm{SO}_{2}$ ) to 
the environment due to fossil fuel usages results in global warming and environmental pollution [2]. Furthermore, high waste of energy in the form of heat due to the low efficiency arising from thermodynamic limits and mishandlings is another disadvantage [3]. In this regard, energy storage is a solution because of their ability to correct the gap between the energy supplied and the energy demand, especially for intermittent energy sources such as solar and wind $[4,5]$.

Many materials are available for use in thermal energy storage systems. However, the selection of suitable material is very much dependent on the specific operating temperature range, the stability of the material for long operation, and the target storage capacity of the system in use [6]. Phase-change materials (PCMs) are one of the most widely used group due to their attractive thermal energy-storage characteristics. They own high energy storage-to-mass ratio as large amounts of latent heat could be stored or released during their solid-liquid phase transitions within almost constant-temperature operating conditions [7-10]. PCMs have been used in different applications including construction, energy recovery, solar energy storage, electronic cooling and domestic buildings [11-13]. Due to the low thermal conductivity of most PCM materials, some investigations have investigated the thermo-physical properties of PCMs $[14,15]$; others were interested in improving the efficiency of the related heat exchangers [1619]. The effort in improving the heat-transfer performance of PCM-based storage systems is seen increasingly advances in recent years. Sadeghi et al. [20] numerically investigated multilayer PCMs in a circular heat exchanger with periodic thermal boundary conditions. They found that for the single-layer unit, using a PCM with high latent heat capacity leads to fluctuating average temperature with low amplitude. Likewise, they found that the multi-layers system in storing latent energy is more applicable. Ghalambaz et al. [21] studied the thermal flow and performance of Nano-Encapsulated Phase Change Materials (NEPCMs) in a cavity. They found that the thermal improvement is extremely based on the non-dimensional fusion 
temperature, and the relative improvement is $10 \%$ compared to the base-fluid. Chamkha et al. [22] Studied the charging process of a nano PCM in a square cavity with a warm cylinder placed in the centre of the cavity with the existence of both single and hybrid nanoparticles. They stated that the solid-liquid interface and the charging rate are mainly influenced by the nanoparticles loading and the thermal conductivity. Moreover, they found that the charging rate is greater when the Fourier number vary between 0 and 0.5 .

Metal foams are one of the superlative techniques in the field of heat transfer enhancement in PCM-based systems [23-27]. Mahdi and Nsofor [9] studied numerically the potential for heattransfer rate enhancement in PCM-based shell-and-tube storage component using multisegment metal foam. They suggested that cascading the pore density in the heat flow direction provides better uniform temperature distribution. Zhu et al. [28] studied the performance of a storage system with $\mathrm{CH}_{3} \mathrm{COONa}$ PCM using various porosities of metal foam and metal fins. It was found that the combination accelerates the melting rate and improves PCM storage performance. Zhang et al. [29] studied the behaviour of the PCM experimentally and numerically. They stated that the composite of paraffin-copper foam improves heat transfer over paraffin wax only due to the high thermal conductivity of the metal foam. Using the metal foam also produces uniform temperature distribution. The numerical model of Zhao et al. [30] improved the efficiency of a high-temperature energy storage system (LHTES) using graphite foam. Using graphite foam reduces the required surface area of heat transfer in the system. Krishnan et al. [31] numerically performed a two-temperature model to assess the efficiency of combining porous medium-PCM using the Darcy-Brinkman equation to study the porous medium effect.

Different heating modes have been used to charge the PCM and store thermal energy which is selected based on the type of application, available source of energy, required charging time and capacity, the melting point of the employed PCM, etc [32]. Different methods have been 
used to charge the PCMs such as heating element, hot fluid flow, heat pipes, heat pumps and renewable energies [33-35]. Moreno et al. [36] experimentally studied on the use of heat pump integrated with a cold TES tank for space cooling application. They compared the use of PCM with water as the storage material and showed that $14.5 \%$ higher capacity of PCM which maintain the indoor temperature $20.65 \%$ longer. However, the charging time for the PCM is 4.55 times higher than water. As a heat storage heater for domestic space heating, Talebizadeh Sardari et al. $[37,38]$ studied the effects of metal foam added to the PCM in a composite porous / PCM to air heat exchanger compared with the PCM-only unit when the PCM was charged by an electrical heating element. They showed the significant advantages of high conductivity porous medium and presented that a uniform output temperature can be gained using a heat transfer enhancement technique by the presence of metal foam inside the PCM. Mettawee and Assassa [39] investigated experimentally the thermal performance of a compact PCM solar collector. Solar energy was stored in the PCM which was then discharged by cold water. They showed a higher rate of heat transfer for a higher thickness of the PCM layer due to the higher effect of natural convection in the domain as the main parameter in spreading the heat in all the domain. Sardari et al. [3] presented a study on heat recovery form domestic radiators using compact PCM unit when the PCM is charged by the hot surface of the radiator. The system was designed to store the excess energy of the radiator for the usage in peak hours.

Heat generation in phase-change materials finds a wide range of applications such as thermal control of electronic components, freezing of biological tissues, and solar thermal energy storage systems. However, few studies have investigated the phase change process inside practical systems with a heat source that is embedded in the PCM [40-42]. Bechiri and Mansouri [43] analytically studied the volumetric heat generation influences on charging and discharging heat transfer of nano-enhanced PCM inside a horizontal cylindrical enclosure. They found that the storage efficiencies are higher and lower than 1 for positive and negative 
heat generation, respectively, and the heat generation influence reduces as liquid fraction increases. Jiji and Gaye [40] analytically analysed charging and discharging heat transfer in a PCM with volumetric energy generation by using a one-dimensional quasi-steady

117 approximation. They developed simple correlations to estimate solidification and melting times

118 based on the heat generation parameter. They presented that the enhanced quasi-steady model 119 is valid for small Stefan numbers.

120 Even though extensive research has been carried out investigating the role of porous foam on improving the functionality of PCMs as storage materials, no previous study adequately 122 investigates the applicability of internal heat-generated porous foam for an improved thermal 123 response of PCM-based latent-heat storage systems. Therefore, the aim of the present work is to fill this gap by numerically investigating the effect of internal uniform heat generation from the porous foam structure during the charging mode of a PCM-in-metal foam latent-heat storage LHS unit. A rectangular container is considered for the heat storage material with the porous medium subjected to Joule heating for uniform internal heat generation. Charging time, heat transfer rate and average temperature are calculated and compared in different cases based on the pore size and the rate of heat generation. Two approaches for internal heat generation are considered. In the first approach, the heat is being uniformly generated inside the domain which can be done by Joule heating of the porous medium and homogeneously propagates to the PCM. The second approach is that the heat is generated from a localized heater in the centre of the system, which is also rarely discussed in the literature. The presence of high conductivity porous medium is commonly used to improve the heat transfer rate in the domain which extremely enhances the heat diffusion inside the domain; however, the opportunity to achieve an efficient thermal energy storage device must rise.

\section{Problem description:}


139 The core of this study is to propose well-performing heater-in-foam latent heat storage (LHS)

140 system by employing the porous structure with volumetric heat generation for superior

141 performance during the energy charging mode. The aim of applying Joule heating is to generate

142 uniform internal heat generation inside the PCM/porous medium composite. This novel method

143 is performed by passing a current through an electrical porous conductor to generate thermal

144 energy. Carbon foam is such a kind of material which can generate heat by Joule heating in

145 addition to the advantage of high thermal conductivity. Therefore, in one hand, due to the

146 presence of the porous medium, the problem of low thermal conductivity of the PCMs is solved

147 and in the other hand, due to the existence of porous medium uniformly in the domain, the heat

148 is generated uniformly and so there is no need to employ many heating elements inside the 149 domain.

150 Similar to the regular electrical heating elements, the heat is generated by passing an electric

151 current through a conductor known as Joule heating. The conductor is considered a porous

152 medium in this study. Therefore, by connecting the porous medium to the electricity, the matrix

153 can generate heat directly. In this study, the volumetric heat generation from the porous

154 structure is studied compared with a localised heater at the centre of the unit. Note that the 155 porosity of porous material is considered $95 \%$ in this study. Therefore, for the volumetric heat 156 generation from the porous medium, the volumetric heat generation is $5 \%$ of the realistic wire 157 volumetric heating rate in the energy equation.

158 The unit is a rectangular cube with the dimensions of $5 \mathrm{~cm} \times 5 \mathrm{~cm} \times 15 \mathrm{~cm}$ filled with PCM 159 embedded in a porous medium. Two walls are considered symmetry (one-quarter of the domain 160 is solved numerically) and the other walls are considered insulated (shown in Fig. 1-a as Case 1610 with heat generation from the matrix). 


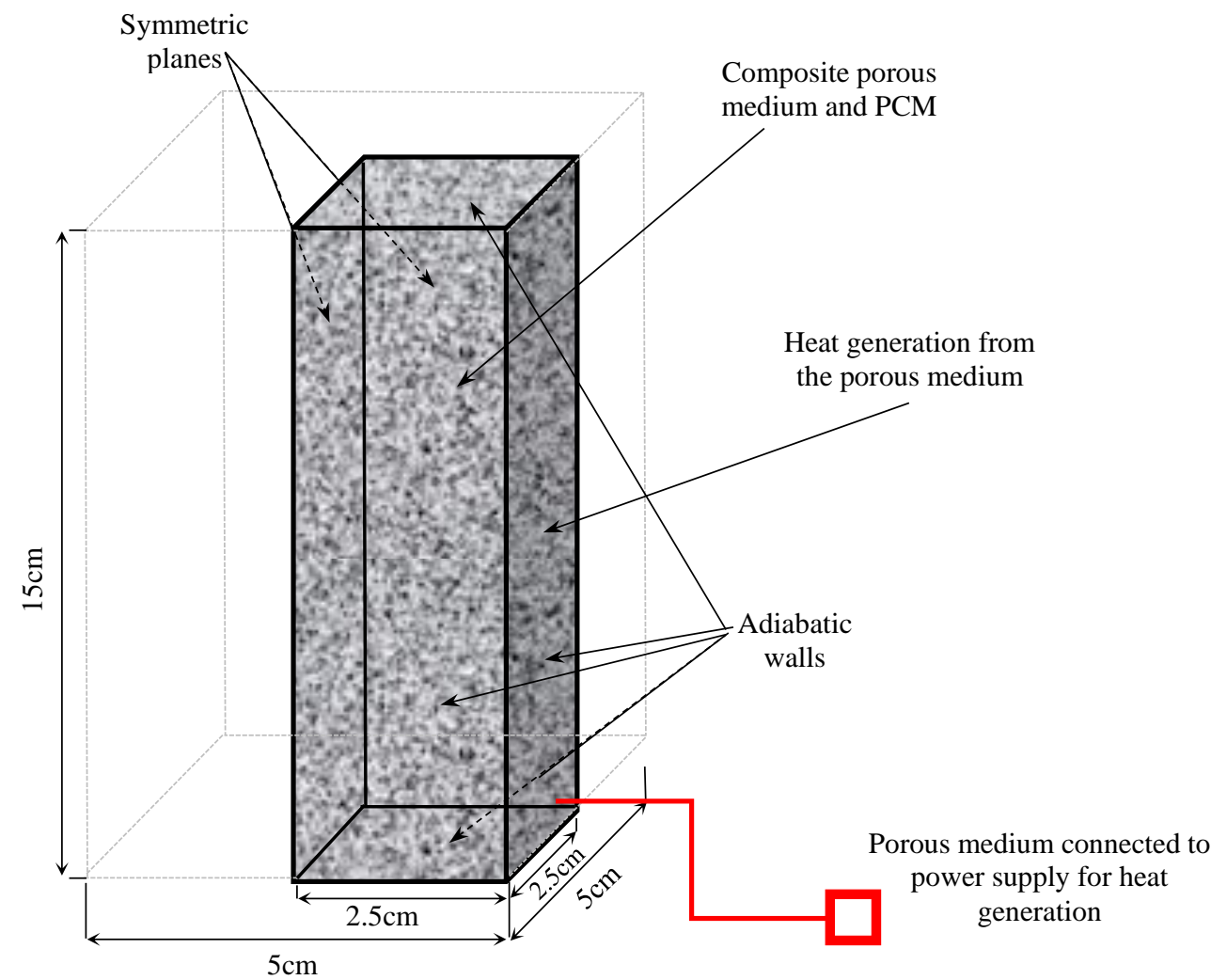

a)

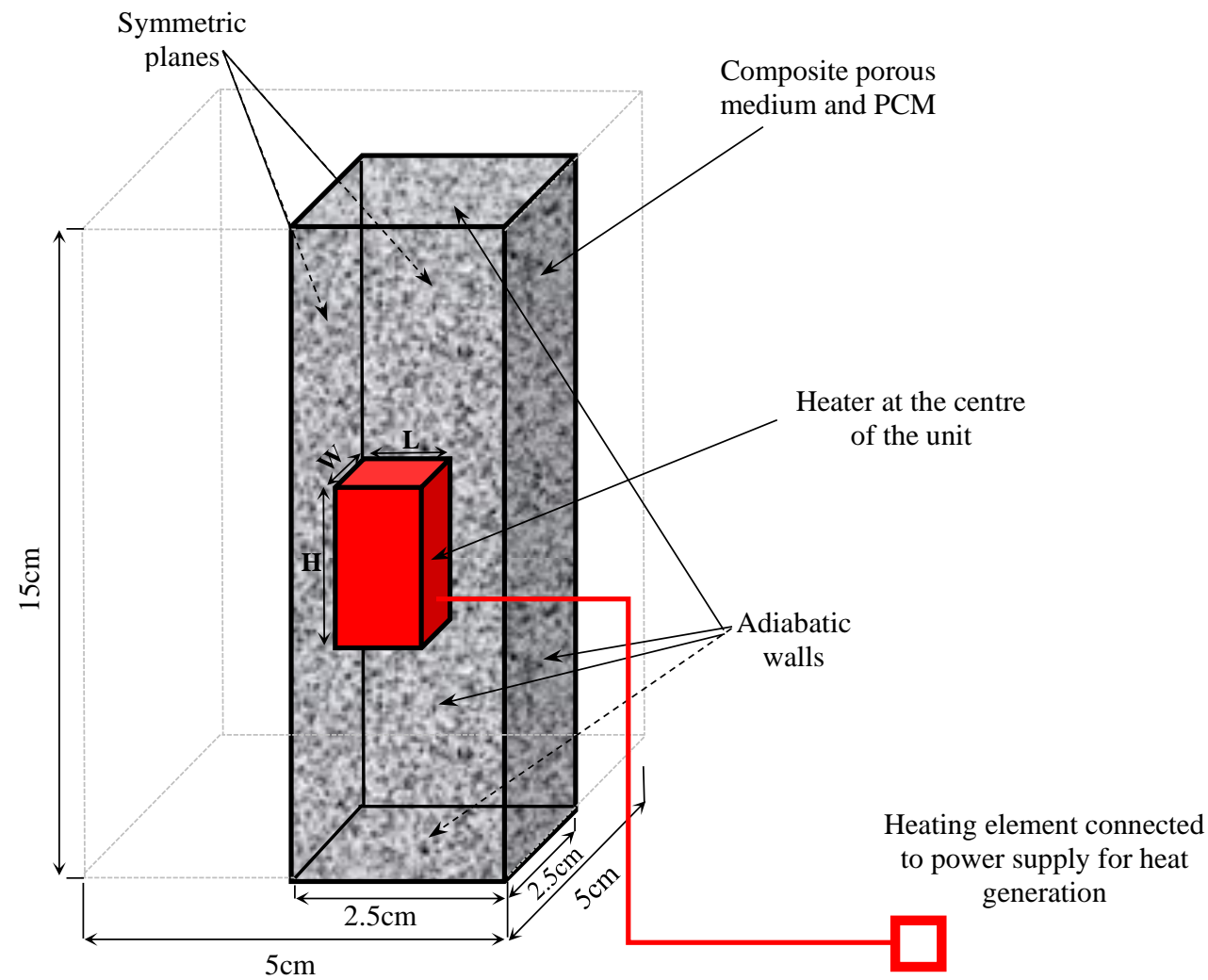

b) 
Fig. 1. The schematic of the studied geometry a) with porous heat generation and b) with a central heating element.

163 For the second studied geometry, a rectangular solid cubic heater is located at the centre of the 164 unit. The volume of the heater is calculated based on the total volume of the porous medium 165 related to the rate of heat generation to have a meaningful comparison. In other words, the volume of the porous medium in the first case is calculated and then the volume of the heater is chosen equal to the volume of the porous medium in the first case. Fig. 1-b shows a schematic of the LHS unit with a localised heating element, and Table 1 presents the dimensions and rates of heat generation of the alternative proposed systems. The dimensions of the heating element in Table 1 is determined as follows: according to the dimensions of the unit, the volume of the porous medium in case 0 is $(15 \mathrm{~cm} \times 2.5 \mathrm{~cm} \times 2.5 \mathrm{~cm} \times 0.05) 4.6875 \mathrm{~cm}^{3}$ for a quarter of the storage unit. 0.05 denotes to the ratio of solid ligament within the porous medium. Therefore, for the central heating element with the height of $2.5 \mathrm{~cm}$ named as case 1, the width (W) of the heating element is $1.369 \mathrm{~cm}$ which is considered equal to the length $(\mathrm{L})$ of the element. The dimensions of the heating elements for the other cases are determined with a similar procedure. Note that in the case of central heater, the PCM domain is also filled with porous medium and the mass of PCM is similar in all the studied cases to have similar storage capacity. Note that in the localised heater cases, the surrounding PCM is embedded in a porous medium; however, the 179 heat is generated only from the central heating element. In Fig. 1-b, only one-quarter of the heater is displayed, and symmetric boundary conditions are used for two surfaces similar to the whole domain. Moreover, the amount of PCM in all the studied cases is constant to have a similar storage capacity in all the proposed cases to have a meaningful comparison.

Note that, as presented in Table 1, Cases 1,2 and 3 have the same heater volume equal to the volume of the porous medium case which has a similar rate of heat generation. For Cases 4 and 
1855 , the volumes of the heating elements are changed based on the rate of heat generation. Cases

1864 and 5 are compared with Case 2 with a similar width and length but different heights when

187 the total heat generated by the heating element (volume $\times$ heat generation rate) is constant.

188

Table 1 The characteristics of the proposed system with a centred heater

\begin{tabular}{lccc}
\hline & $\mathrm{H}(\mathrm{cm})$ & $\mathrm{W}=\mathrm{L}(\mathrm{cm})$ & Heat generation rate $\left(\mathrm{kW} / \mathrm{m}^{3}\right)$ \\
\hline Case 1 & 2.5 & 1.369 & 100 \\
Case 2 & 5 & 0.968 & 100 \\
Case 3 & 10 & 0.685 & 100 \\
Case 4 & 2.5 & 0.968 & 200 \\
Case 5 & 10 & 0.968 & 50 \\
\hline
\end{tabular}

189

190 RT-35 (RUBITHERM) is considered as the PCM with the physical properties presented in 191 Table 2.

192

Table 2 The properties of RT 35 [44].

\begin{tabular}{cc}
\hline Property & RT35 \\
\hline Liquidus/Solidus temperature $\left({ }^{\circ} \mathrm{C}\right)$ & $309 / 302$ \\
Latent heat of fusion $(\mathrm{kJ} / \mathrm{kg})$ & 170 \\
Specific heat $(\mathrm{kJ} / \mathrm{kgK})$ & 2 \\
Expansion coefficient $(1 / \mathrm{K})$ & 0.001 \\
Thermal Conductivity $(\mathrm{W} / \mathrm{mK})$ & 0.2 \\
Viscosity $(\mathrm{Pas})$ & 0.023 \\
Density $\left(\mathrm{kg} / \mathrm{m}^{3}\right)$ & 815 \\
\hline
\end{tabular}

193

194 Note that the initial temperature of the PCM is considered equal to $292 \mathrm{~K}$.

195

196 


\section{Mathematical description}

198

$$
\begin{aligned}
& \frac{\partial \rho}{\partial t}+\nabla \cdot \rho \vec{V}=0 \\
& \frac{\rho}{\varepsilon} \frac{\partial \vec{V}}{\partial t}+\frac{\rho}{\varepsilon^{2}}(\vec{V} \cdot \nabla) \vec{V}=-\nabla P+\frac{\mu}{\varepsilon}\left(\nabla^{2} \vec{V}\right)-\rho_{r e f} \beta \varepsilon\left(T-T_{r e f}\right) \vec{g}-\vec{S}-\vec{F}
\end{aligned}
$$

For the PCM

$$
\frac{\partial \varepsilon \rho_{f} C_{p, f} T}{\partial t}+\nabla\left(\rho_{f} C_{p, f} \vec{V} T\right)=\nabla\left(k_{f e} \nabla T\right)-S_{L}-h_{s f} A_{s f}\left(T_{f}-T_{s}\right)
$$

For the porous medium:

$$
(1-\varepsilon) \rho_{s} C_{p, s}\left(\frac{\partial T_{s}}{\partial t}\right)=\nabla\left(k_{s e} \nabla T_{f}\right)-h_{s f} A_{s f}\left(T_{s}-T_{f}\right)-S_{g}
$$

In the momentum equation, to consider the effect of natural convention and buoyant flow, the Boussinesq approximation is employed. Furthermore, in Eq. (4), for the case of heat generation in a separate solid zone.

$k_{f e}$ and $k_{s e}$ should be calculated for the PCM and porous medium separately which are 


$$
k_{e f f}=\frac{1}{\sqrt{2}\left(R_{A}+R_{B}+R_{C}+R_{D}\right)}
$$

214 where

$$
\begin{aligned}
& R_{A}=\frac{4 \sigma}{\left(2 e^{2}+\pi \sigma(1-e)\right) k_{s}+\left(4-2 e^{2}-\pi \sigma(1-e)\right) k_{f}} \\
& R_{B}=\frac{(e-2 \sigma)^{2}}{(e-2 \sigma) e^{2} k_{s}+\left(2 e-4 \sigma-(e-2 \sigma) e^{2}\right) k_{f}} \\
& R_{C}=\frac{\sqrt{2}-2 e}{\sqrt{2} \pi \sigma^{2} k_{s}+\left(2-\sqrt{2} \pi \sigma^{2}\right) k_{f}} \\
& R_{D}=\frac{2 e}{e^{2} k_{s}+\left(4-e^{2}\right) k_{f}}
\end{aligned}
$$

215 where $e=0.16$ and

$$
\sigma=\sqrt{\frac{\sqrt{2}\left(2-\left(\frac{3 \sqrt{2}}{4}\right) e^{3}-2 \varepsilon\right)}{\pi(3-2 \sqrt{2} e-e)}}
$$

216 and

$$
\begin{aligned}
& k_{f e}=k_{e f f} \mid k_{s=0} \\
& k_{s e}=k_{e f f} \mid k_{f=0}
\end{aligned}
$$

217 The source term in the momentum equation is given as [48]:

$$
\vec{S}=A_{m} \frac{(1-\lambda)^{2}}{\lambda^{3}+0.001} \vec{V}
$$

218 where $A_{m}$ is $10^{5}$ [49-51]. Additionally, $\lambda$ is defined as [52]:

$$
\lambda=\frac{\Delta H}{L}=\left\{\begin{array}{cc}
0 & \text { if } T<T_{\text {Solidus }} \\
1 & \text { if } T>T_{\text {Liquidus }} \\
\frac{T-T_{\text {Solidus }}}{T_{\text {Liquidus }}-T_{\text {Solidus }}} & \text { if } T_{\text {Solidus }}<T<T_{\text {Liquidus }}
\end{array}\right\}
$$

219 where $\Delta H$ varies between zero for the solid-state and $L$ for the liquid state.

220 The body force in the momentum equation is defined as: 


$$
\vec{F}=\left(\frac{\mu}{K}+\frac{\rho C|\vec{V}|}{\sqrt{K}}\right) \vec{V}
$$

221 In this equation, $K$ is the permeability given as [53]:

$$
K=0.00073 d_{p}^{2}(1-\varepsilon)^{-0.224}\left(\frac{d_{l}}{d_{p}}\right)^{-1.11}
$$

222 and $\mathrm{C}$ is the inertial coefficient given as [53]:

$$
C=0.00212(1-\varepsilon)^{-0.132}\left(\frac{d_{l}}{d_{p}}\right)^{-1.63}
$$

223 where $d_{l}$ is the ligament or cell diameter is given as:

$$
d_{l}=1.18 d_{p} \sqrt{\frac{1-\varepsilon}{3 \pi}}\left(\frac{1}{1-e^{-(1-\varepsilon) / 0.04}}\right)
$$

$224 d_{p}$ is the pore size given as:

$$
d_{p}=0.0254(m) / \omega
$$

225 Note that $\omega$ is the pore density with the unit of PPI means pores per inch.

226 In Eq. (3), $S_{L}$ is given as [4]:

$$
S_{L}=\frac{\partial \varepsilon \rho \lambda L}{\partial t}+\nabla(\rho \vec{V} \lambda L)
$$

227 To calculate the local heat transfer between the porous medium and PCM, the porous structure 228 is usually considered as cylinders and the laminar flow of liquid PCM in porous structure is 229 considered similar to the flow around a cylinder [54]. Therefore, the interstitial heat transfer 230 coefficient is calculated, for the appropriate range of Reynolds number, as [37, 44]:

$$
h_{s f}=0.76 \operatorname{Re}_{d}^{0.4} \operatorname{Pr}^{0.37} k_{f} / d_{l} \text { for } \quad 0<R e_{d} \leq 40
$$

231 where

$$
R e_{d}=\rho_{p c m}\left(\sqrt{\sum_{i=1}^{3} u_{i}^{2}}\right) d_{l} /\left(\varepsilon \mu_{f}\right)
$$


and $\boldsymbol{A}_{\boldsymbol{s} f}$ is the specific surface area of the porous medium given as:

$$
A_{s f}=\frac{3 \pi d_{l}\left(1-e^{-(1-\varepsilon) / 0.04}\right)}{0.59 d_{p}{ }^{2}}
$$

233 Note that before the PCM starts to melt, there is no convection heat transfer inside the pores. It

234 can be found in Eq. (21) when $h_{s f}$ is zero since $R e_{d}$ is zero based on Eq. (22).

\section{Numerical process and code verification}

237 The equations governed on the problem are solved using ANSYS-FLUENT utilizing a UDF 238 (User-defined functions) to determine $h_{s f}$. A detailed discretion of the numerical process is 239 discussed in Ref. [46, 47]. For mesh analysis, different cases are studied considering a higher 240 mesh density in y-direction due to the presence of gravity as well as an equal number of nodes

241 for both $\mathrm{x}$ and $\mathrm{z}$-directions. Table 3 presents the melting time for both cases of internal heat 242 generation from the porous medium and localised heater (Case 1 in Table 1) for the heat 243 generation rate of $100 \mathrm{~kW} / \mathrm{m}^{3}$. The porosity and pore density of the porous medium are 244 considered $95 \%$ and 30 PPI, respectively. The results show that after the number of 37,500 245 nodes $(60 \times 25 \times 25)$, there is no considerable variation in the results. As presented, the difference 246 between the melting times for different cell numbers for the case of heat generation from the 247 porous medium is negligible due to the uniform heat generation distribution in the domain.

Table 3 Mesh independence analysis for different number of cells

\begin{tabular}{ccc}
\hline & $\begin{array}{c}\text { Heat generation from the porous } \\
\text { medium }\end{array}$ & $\begin{array}{c}\text { Localised heat generation from the } \\
\text { heating element }\end{array}$ \\
\hline Number of cells & Melting time (min) & 1004.2 \\
25000 & 836.3 & 1015.4 \\
37500 & 837.5 & 1016.3 \\
50000 & 837.7 & \\
\hline
\end{tabular}


251 The size of the time step is $0.5 \mathrm{~s}$ and there is no variation seen by reducing the time step size 252 down to $0.25 \mathrm{~s}$. The schematic of the entire computational mesh, as well as the front and top 253 views, is shown in Fig. 2.
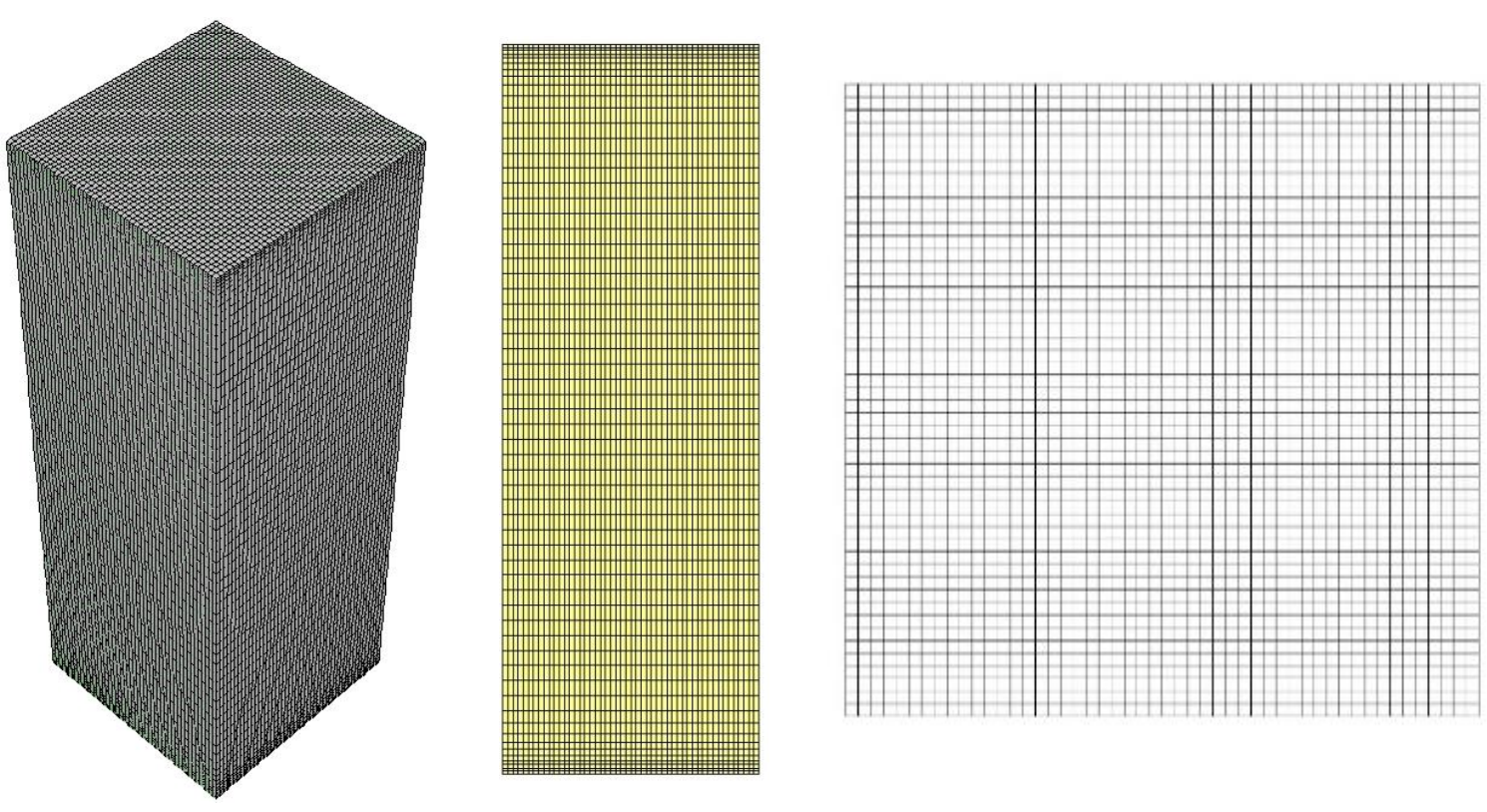

Fig. 2. The computational domain at different views.

254 To verify the method, a rectangle heat storage unit with the dimensions of $200 \times 120 \times 25 \mathrm{~mm}$ is 255 modelled which is studied experimentally by Zhao et al. [8, 55] and numerically using the 256 thermal non-equilibrium model by Liu et al. [56]. The LHS unit includes a combination of 257 PCM (RT-58) with metal foam with $95 \%$ porosity and 10 PPI pore density considering a heat 258 flux boundary condition of $1600 \mathrm{~W} / \mathrm{m}^{2}$ for the bottom surface and convection for the other 259 boundaries. The temperature located at the centre at the height of $8 \mathrm{~mm}$ is presented in Fig. 3 260 shows an excellent agreement with both numerical and experimental analysis. The maximum 261 differences between the present work and the work of Liu et al. [56] and Zhao et al. [8] are 6\% and $3 \%$, respectively. 


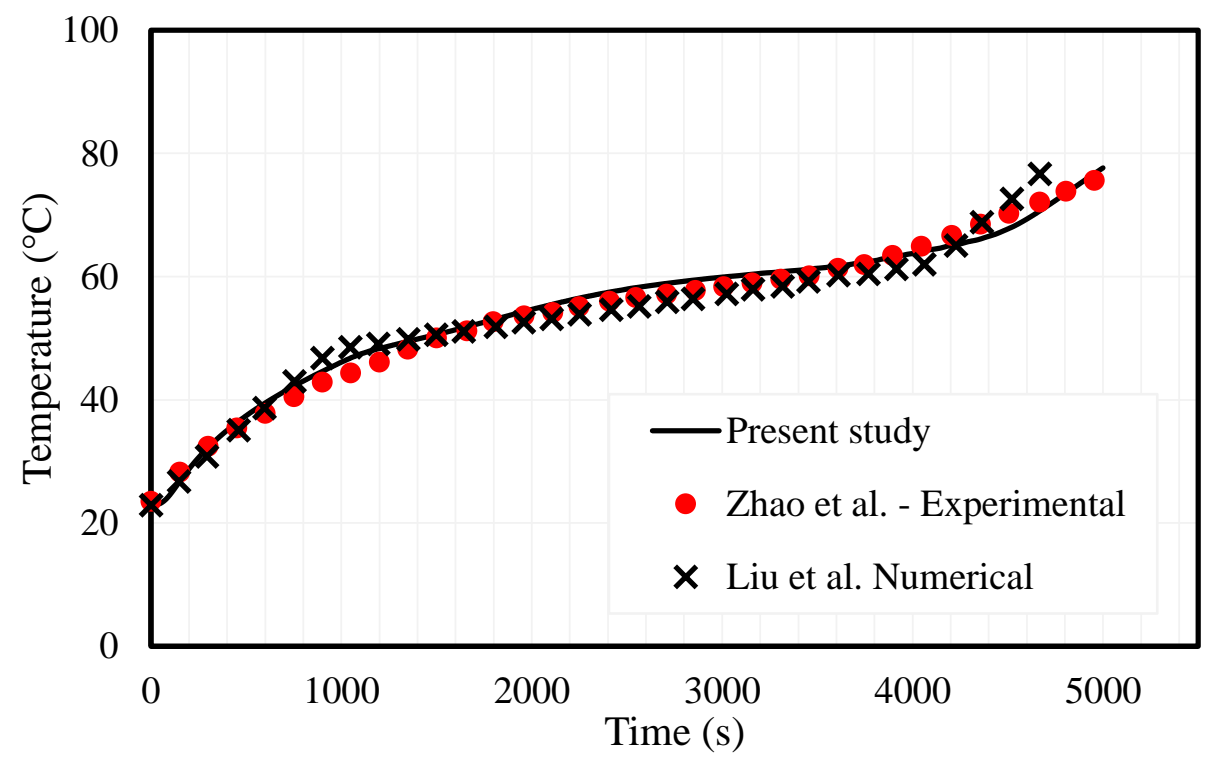

Fig. 3. The validation study of a rectangular MFLHS system compared with experimental data of Zhao et al. [55] and numerical study of Liu et al [56]

\section{Results and discussion}

266 In this section, the internal heat generation from the porous structure is firstly discussed, then

267 the results of a localised heater are analysed after studying the effective parameters.

\subsection{Heat generation from the porous structure}

270 By volumetric heat generation from the porous medium, the temperature and liquid fraction 271 field distribution are uniform and in an identical time, all the field has an almost constant 272 temperature and liquid fraction. Fig. 4 displays the variation of PCM mean temperature and

273 liquid fraction as well as the mean temperature of the porous medium in terms of time for the 274 PCM-in- foam LHS unit. Note that the porosity and pore density is selected equal to $95 \%$ and $27530 \mathrm{PPI}$, respectively, with the heat generation rate of $100 \mathrm{~kW} / \mathrm{m}^{3}$. When the temperature rises 276 from $302 \mathrm{~K}$, the melting process starts after almost 115 minutes. At the time of 837.5 minutes, 277 when the temperature is almost 309 , the liquid fraction reaches to one and the PCM melts 
completely. During the phase change, the PCM temperature rises with a low rate due to placing

279 PCM in the latent heat section after the beginning sharp increase. The temperature of the porous

280 medium is virtually equal to the PCM since the foam generates heat directly and the number

281 of pores is high (30 PPI). A negligible temperature difference exists between the PCM and

282 porous temperatures. The reason is due to the uniform heat generation in the domain which

283 lessens the difference between the temperature of PCM and porous medium. As shown in Fig.

2841 , the heat is generated uniformly in the entire domain from the surface of the pores in the

285 porous medium and due to the small volume of the pores, the temperatures of the PCM and

286 porous medium are almost the same. It should be noted that due to the uniform distribution of

287 liquid fraction and temperature, the contour plots are uniform and at an identical time, all the domain has an almost constant liquid fraction and temperature due to the uniform heat generation inside the domain.

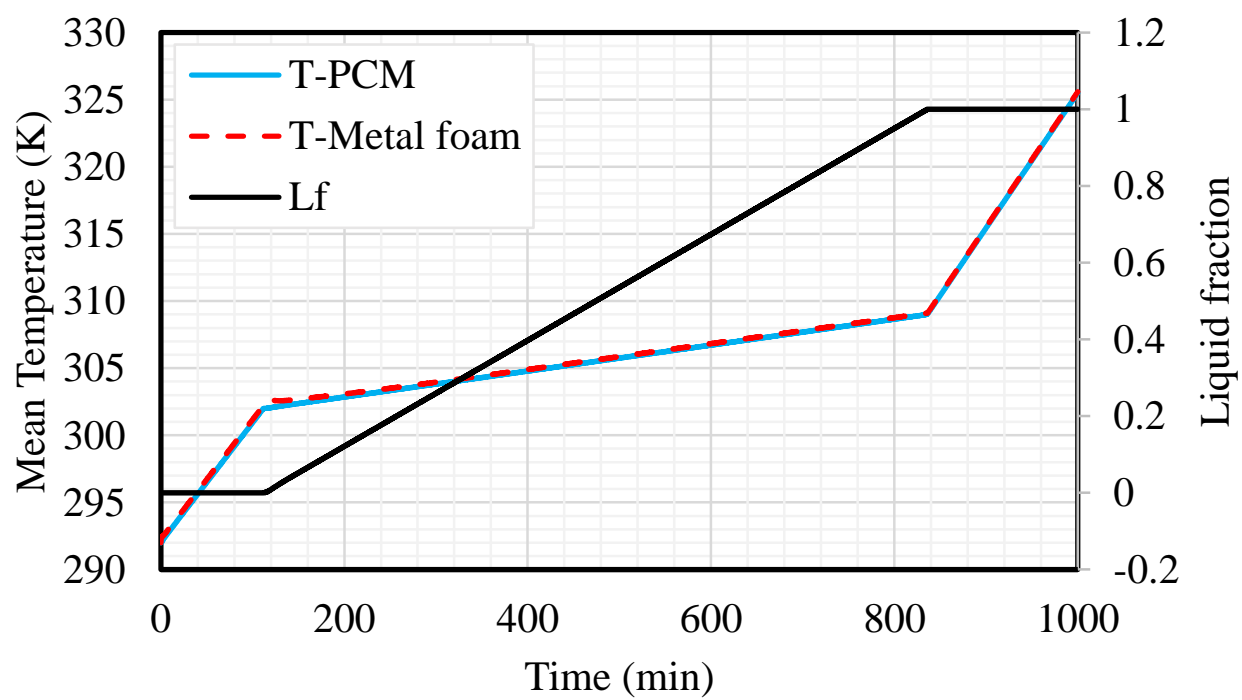

Fig. 4. The variation of PCM mean temperature and liquid fraction as well as porous temperature for the LHS unit with $\varepsilon=0.95 \%, \omega=30$ PPI and $\dot{q}=100 \mathrm{~kW} / \mathrm{m}^{3}$

292 Fig. 5 illustrates the variation of temperature at three different points with various heights at 293 the centre of the system. As shown, the temperatures at different heights are similar. The main 
reason is the proposed charging method which is uniform heat generation from the porous medium in the system. Thus, because of uniform internal heat generation, the temperatures are almost similar in the domain. Furthermore, due to the presence of high conductivity porous medium and thus high rate of heat transfer, the temperature is uniform in all the domain. Consequently, all point has equal temperatures. These are also the reasons for the equilibrium condition between the PCM and porous medium shown in Fig. 4. After a sharp temperature enhancement, during the phase change process, the temperature rises to a lower rate and then increases sharply.

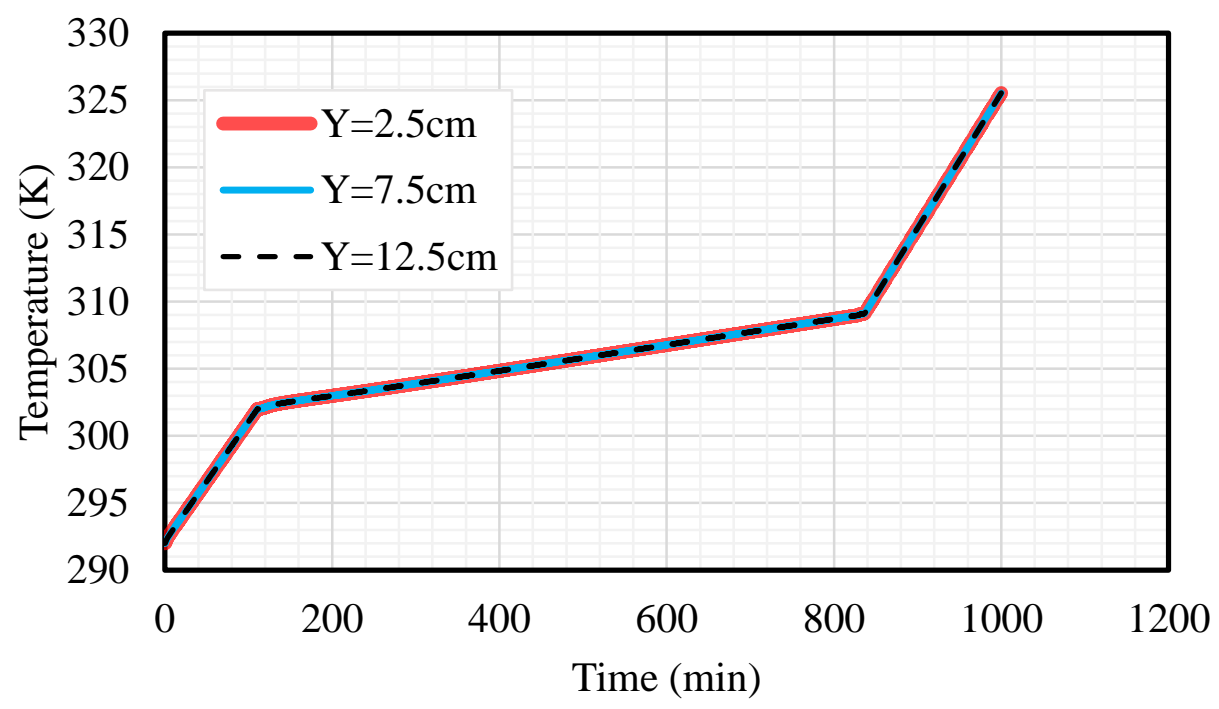

Fig. 5. The variation of PCM temperature at different points for the LHS unit with $\varepsilon=0.95 \%$, $\omega=30$ PPI and $\dot{q}=100 \mathrm{~kW} / \mathrm{m}^{3}$

\subsubsection{Influence of volumetric heat generation rate}

The influence of the volumetric heat generation rate on the PCM mean temperature and liquid fraction are illustrated in Figs. 6-a and 6-b, respectively. By applying a higher heat generation rate, the PCM melts in a shorter time expectedly. During the charging process, the temperature is almost constant (vary between solidus and liquidus temperatures) and then rises sharply. For a higher heat generation rate, a higher slope of the temperature rising line is achieved during 
310 the phase change. At the time when the PCM melts entirely for the heat generation rate of 200

$311 \mathrm{~kW} / \mathrm{m}^{3}$, the mean PCM temperature is almost $400 \mathrm{~K}$ for the case of $400 \mathrm{~kW} / \mathrm{m}^{3}$. Note that since

312 the heat generations are different for various cases, as mentioned, the melting times are also

313 varied, and the simulations are terminated when the PCM melts completely. Therefore, in Fig.

$3146-\mathrm{a}$, the times of the simulations are varied.

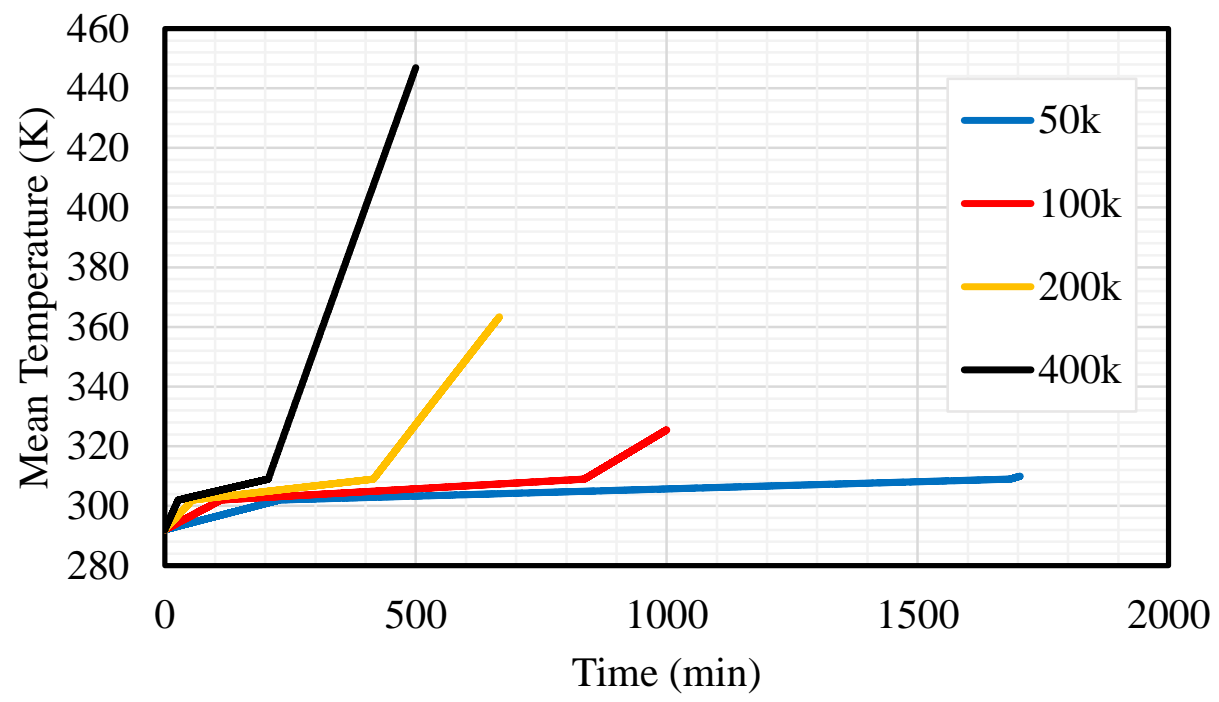

a)

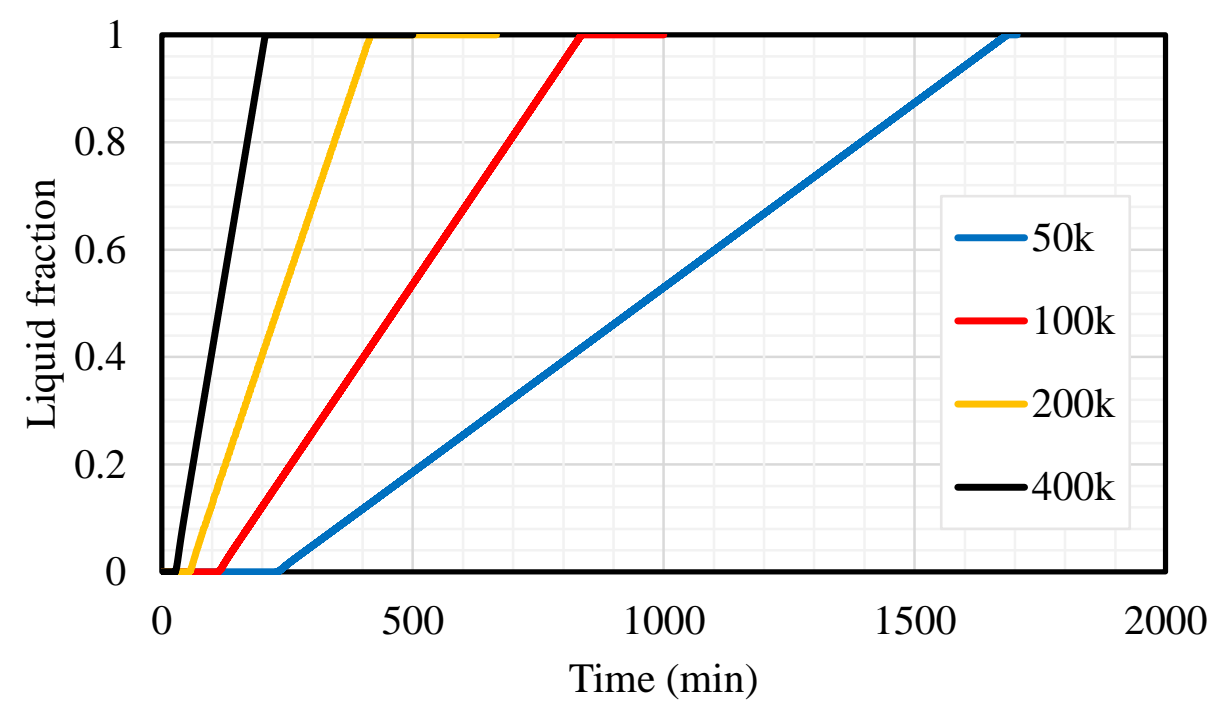

b) 
Fig. 6. The variation of a) PCM mean temperature and b) liquid fraction Case 0 for different heat generations rates

317 Table 4 presents the melting time and the time-saving percentage compared with the heat 318 generation rate of $100 \mathrm{~kW} / \mathrm{m}^{3}$ for different rates of heat generation. By enhancing the rate of 319 heat generation, the melting time reduces with a constant rate approximately. By doubling the 320 heat generation rate, the melting time reduces by almost $50 \%$. The reason is due to the uniform 321 distribution of heat generation inside the domain as a result of a homogeneous porous medium.

322 This is also can be seen in Fig. 6-b. This can be also explained according to the dimensionless 323 numbers of Fourier number $\left(\frac{\alpha t}{H^{2}}\right)$ and Stefan number $\left(\frac{C_{p} q_{w} \mathrm{H}}{k L}\right)$ and Rayleigh number $324\left(\frac{\rho^{2} g \beta C_{p} q_{w} \mathrm{H}^{4}}{k^{2} \mu}\right)$ where $q_{w}$ is defined as the heat flux of the heat source. The non-dimensional 325 analysis was performed in the literature for different cases and the results have shown that the 326 liquid fraction is varied as a function of Fourier, Stefan and Rayleigh numbers [4, 46, 51, 57$32760]$. Since natural convection is negligible in the existence of metal foam, the Rayleigh number 328 variation does not affect the liquid fraction significantly [46]. In the proposed system in this study, by doubling the heat generation rate and considering all the other parameters constant, 330 Stefan number also doubles results in a lower melting time by almost $50 \%$ as presented in 331 Table 4. Note that the liquid fraction and melting time is proportional directly with the Stephan 332 number $[4,46,51,57,60]$.

Table 4 The melting time and the time-saving percentage for Case 0 for different heat generation rates compared with the heat generation rate of $100 \mathrm{~kW} / \mathrm{m}^{3}$

\begin{tabular}{ccc}
\hline Heat generation rate $(\mathrm{kW} / \mathrm{m} 3)$ & Melting time $(\mathrm{min})$ & Timesaving \\
\hline 50 & 1685.92 & -101.30 \\
100 & 837.50 & 0.00 \\
200 & 417.00 & 50.20
\end{tabular}


336 Fig. 7 displays the transient effect of pore density on the PCM mean temperature (Fig. 7-a) and 337 the liquid fraction (7-b) for Case 0 with $\dot{Q}=100 \mathrm{~kW} / \mathrm{m}^{3}$. By changing the pore density of the porous medium, the size of pores changes results in a different inertial coefficient and permeability of the porous medium in the momentum equation. As shown in Fig. 7-a, a negligibly higher temperature is achieved using a higher pore density resulting in a negligibly

341 lower melting time (Fig. 7-b). The larger pore density enhances the solid-liquid interfacial 342 surface area results in a higher effective thermal conductivity. However, increasing the pore 343 density reduces the permeability which results in the lower natural convection effect. 344 Therefore, it is a trade-off process of PCM melting in different pore densities [61]. In the 345 proposed system, the main reason for the negligible effect of pore density is the heat generated 346 from the porous medium for the charging process. Due to the equal amount of uniform heat 347 generation from the porous medium for different pore densities and due to the high effect of 348 heat generation in the proposed system, the effect of pore density is negligible. However, as 349 shown, by using a higher pore density, the distribution of the heat generated in the domain is more uniform helping the melting process. 


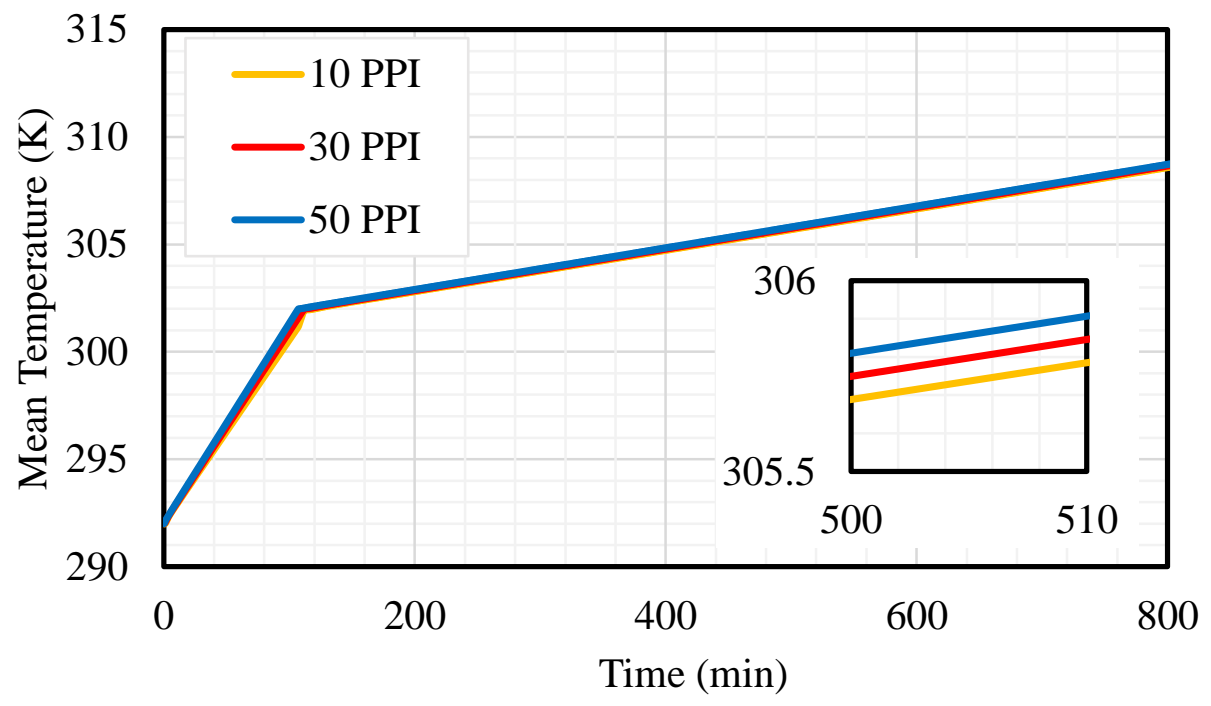

a)

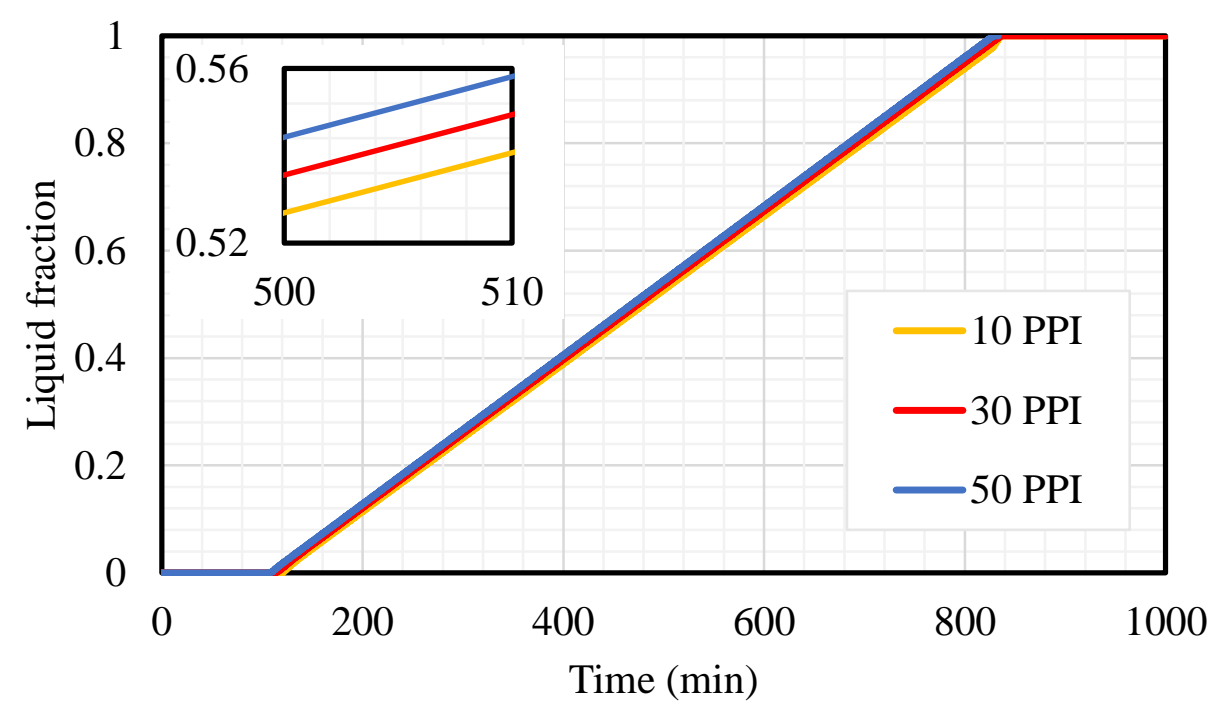

b)

Fig. 7. The variation of a) PCM mean temperature and b) liquid fraction for Case 0 with $\dot{Q}=100 \mathrm{~kW} / \mathrm{m}^{3}$ for different pore densities

354 To show the advantages of the internal heat generation, a PCM-in-metal foam LHS unit with a 355 heater (the heater was designed as a solid block (without porous medium and PCM) and defined 
to give a constant heat flux) at the centre with a similar rate of heat generation is simulated. As

357 mentioned, the dimensions of the heater are selected based on the volume of porous structure

358 in Case 0. Fig. 8 shows the contour plot of PCM temperature (Fig. 8-a) and the liquid fraction

359 (Fig. 8-b) at 10000s time intervals for the Case 1 in Table 1 at the middle section of the unit. It

360 should be noted that in the liquid fraction contours, the liquid fraction is varied between 0 and

1. When the liquid fraction is 1 , it means that the PCM is completely in the liquid state and when it is 0 , the PCM is in the solid-state. Between 0 and 1 , the PCM is placed in the mushy zone.

364 In Fig. 8, the height and length of the heater are $2.5 \mathrm{~cm}$ and $1.369 \mathrm{~cm}$, respectively. The heat 365 transfers out in the domain from the central heating element toward the walls. The temperature of the heater increases with time and when it reaches the solidus PCM temperature, melting starts. Due to the presence of metal foam inside the domain, a uniform temperature distribution can be seen as discussed in [47]. As shown in Fig. 8-b, a higher liquid fraction occurs around the heater due to the higher temperature of the heater compared with the other parts of the unit;

370 however, due to the effect of surrounding metal foam, the liquid fraction enhances in all the domain but with a higher rate around the heater.

372

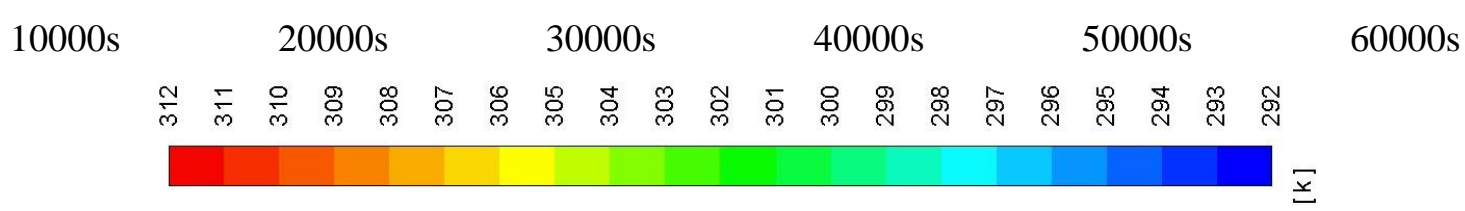



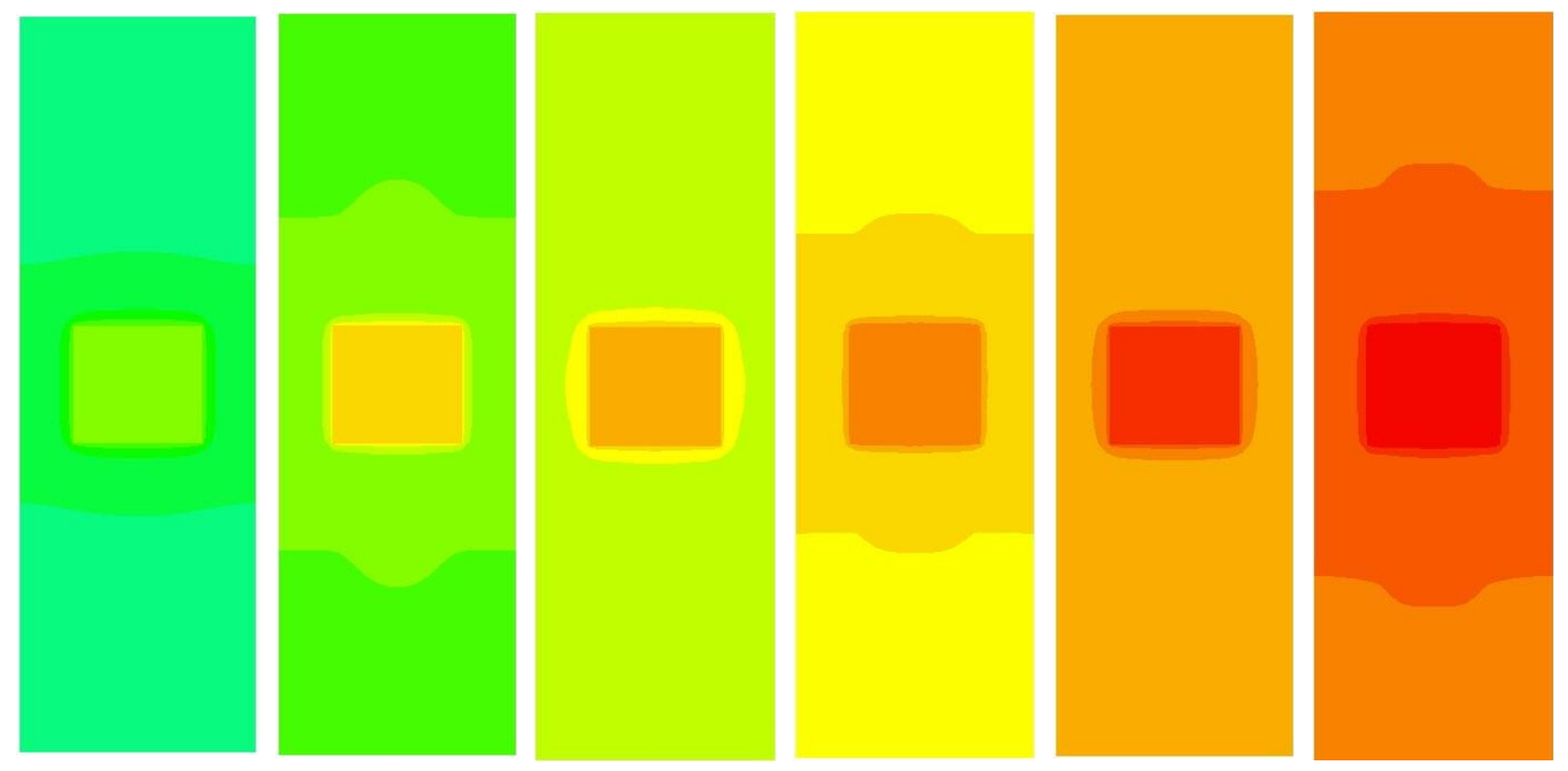

a)

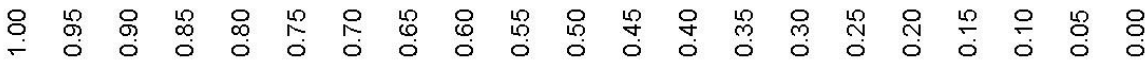
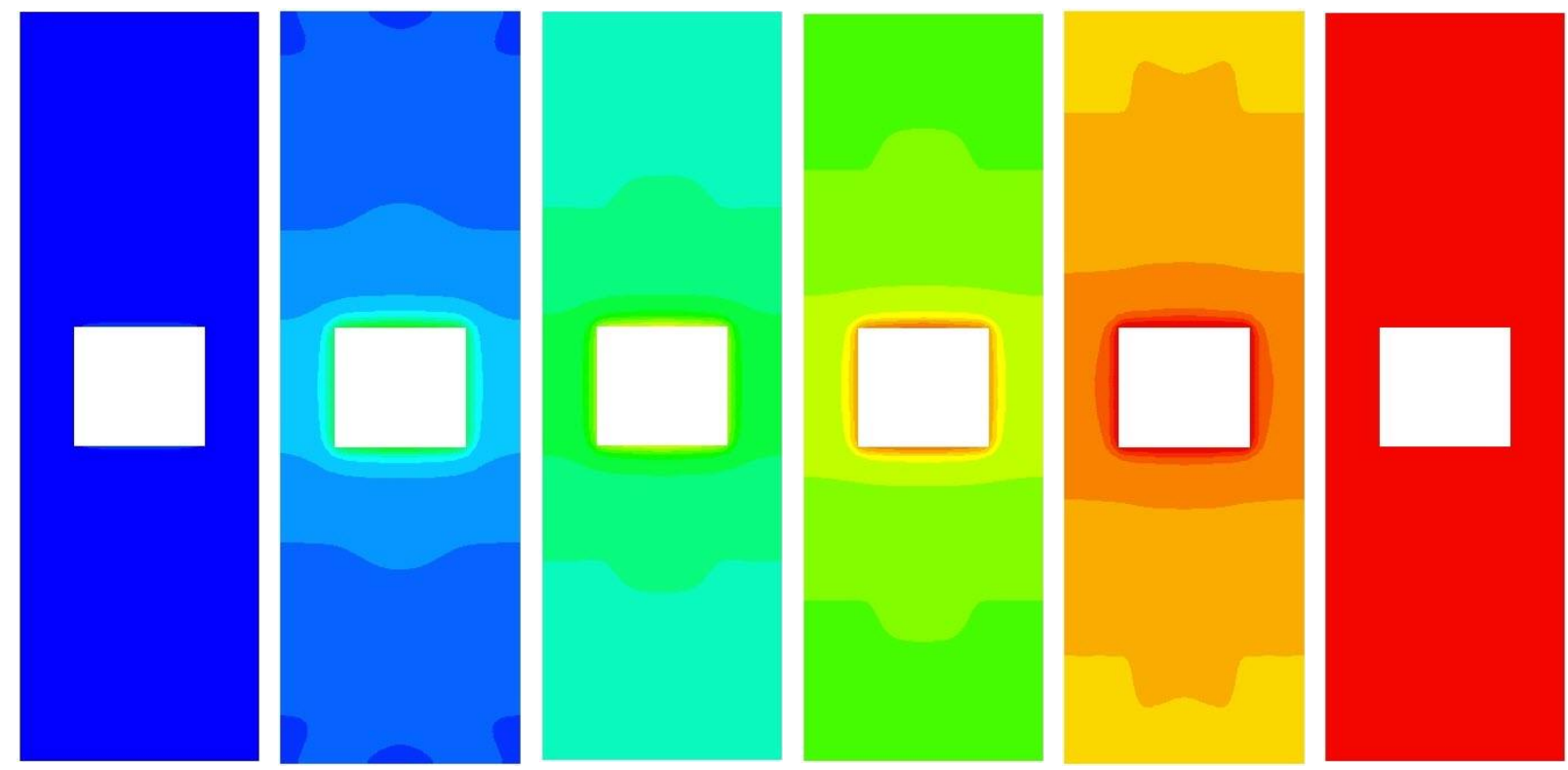

b)

Fig. 8. The distribution of a) temperature and b) liquid fraction at various times for the system using a centred heater with $\varepsilon=0.95 \%, \omega=30$ PPI and $\dot{Q}=100 \mathrm{~kW} / \mathrm{m}^{3}$

374 The total melting time is 1015.4 minutes for Case 1 , which is $17.5 \%$ higher than that compared

375 with the case of an internal heat generation even with the presence of a high conductivity porous medium. Note that it is expected that by using a higher area of the heat exchanger with constant 
heat generation rate, the heat is transferred quicker from the heating element to the PCM and therefore it has a high impact on the melting rate. In the case of internal heat generation from the porous medium, a heat source with a higher surface area is employed results in a shorter melting time. Therefore, the novel introduced method of uniform heat generation using Joule heating is proven as a more advantageous method for reducing the charging time compared with using metal foam with a separate heater.

\subsubsection{Influence of heating element dimension at a constant heat generation rate}

As mentioned in Table 1, for a constant heat generation rate of $100 \mathrm{~kW} / \mathrm{m}^{3}$, three different sizes for the heating element are studied with the same volume compared with the case of internal heat generation from the porous medium. Fig. 9 shows the effect of the central heating element on the mean temperature of the PCM, respectively. For all units with a central heating element, lower mean temperature of the PCM can be seen compared with the case of Joule heating from the porous medium. For a lower height, a higher mean temperature is achieved results in a lower melting time. The relatively high flow-resistant forces due to the existence of the porous medium largely affect the convective flow of liquid PCM and make the role of natural convection to be almost negligible. Therefore, the reason for the advantage of case 1 over other cases is only related to the dimensions of the unit. It means that due to the effect of the porous medium which results in suppressing the effect of natural convection, conduction is the main mechanism for heat transfer and therefore the direction of the heating element is not important. In other words, it is not important that which edge of the heating element is in the gravity direction. Therefore, the heating element with a more uniform distribution in the domain related to the dimensions of the storage unit can be more effective for heat generation. Therefore, case 1, which the heating element is more uniform in all directions according to Table 1 , has the best performance higher temperature and a higher melting rate compared with other studied 
cases with central heating elements. The melting time of case 1 is almost $2 \%$ and $3 \%$ less than

403

404

405

406

407

408

409

cases 2 and 3, respectively. It should be noted that although the differences are small but have an effect on improving the performance of the LHS systems. Furthermore, considering the studied effect in a larger size of an LHS system, the effect can be more pronounced results in a higher energy saving. Note that, in general, due to the presence of metal foam in the system, the temperature difference in the systems with a central heating element with different dimensions is very small.

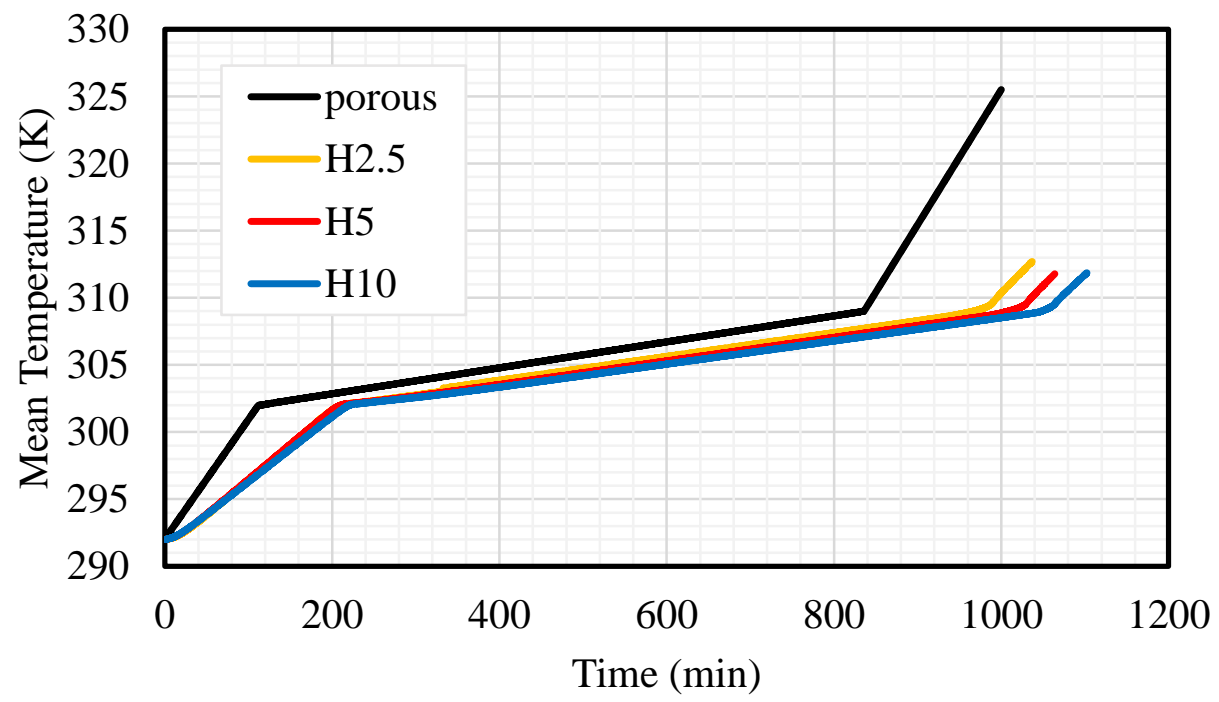

Fig. 9. The variation of the mean PCM temperature in terms of time for the system with a central heating element with different sizes with $\varepsilon=0.95 \%, \omega=30$ PPI and $\dot{Q}=100 \mathrm{~kW} / \mathrm{m}^{3}$

413 To understand the effect of heating element size more clearly, Figs. 10-a and 10-b illustrate the 414 mean temperature and liquid fraction of PCM for Case 2 in Table 2 compared with Cases 4 and

4155 where the horizontal area of the heating element is considered constant and the height is 416 changed based of the rate of heat generation. Note that in all cases, the amount of heat 
417 generation from the heat source is the same. As shown, the unit with a lower height and higher 418 heat generation rate shows a higher mean temperature and results in a lower melting time.

419 Because of the high conductivity porous medium in the domain, as mentioned, the effect of 420 heating element dimensions is suppressed. Therefore, a case with a higher heat generation rate 421 shows better results. According to the hear generation rate, the volumetric heat source power 422 is identical for all cases of 2, 4 and 5; however, for the case with higher heat generation rate 423 per volume, the heat transfers faster inside the porous medium to due to a higher heat generation 424 rate and as a result penetrates faster in all the domain. Thus, in an identical time, the temperature 425 in the case with higher heat generation rate (case 4) is higher as shown in Fig. 10. The melting 426 time for case 4 is $4.7 \%$ and $14.7 \%$ higher than that for cases 2 and 5, respectively. The results 427 of this section show that, for an identical volumetric heat source power, the rate of heat 428 generation per volume is more pronounced than the surface area of the heating elements due to 429 the presence of the porous medium.

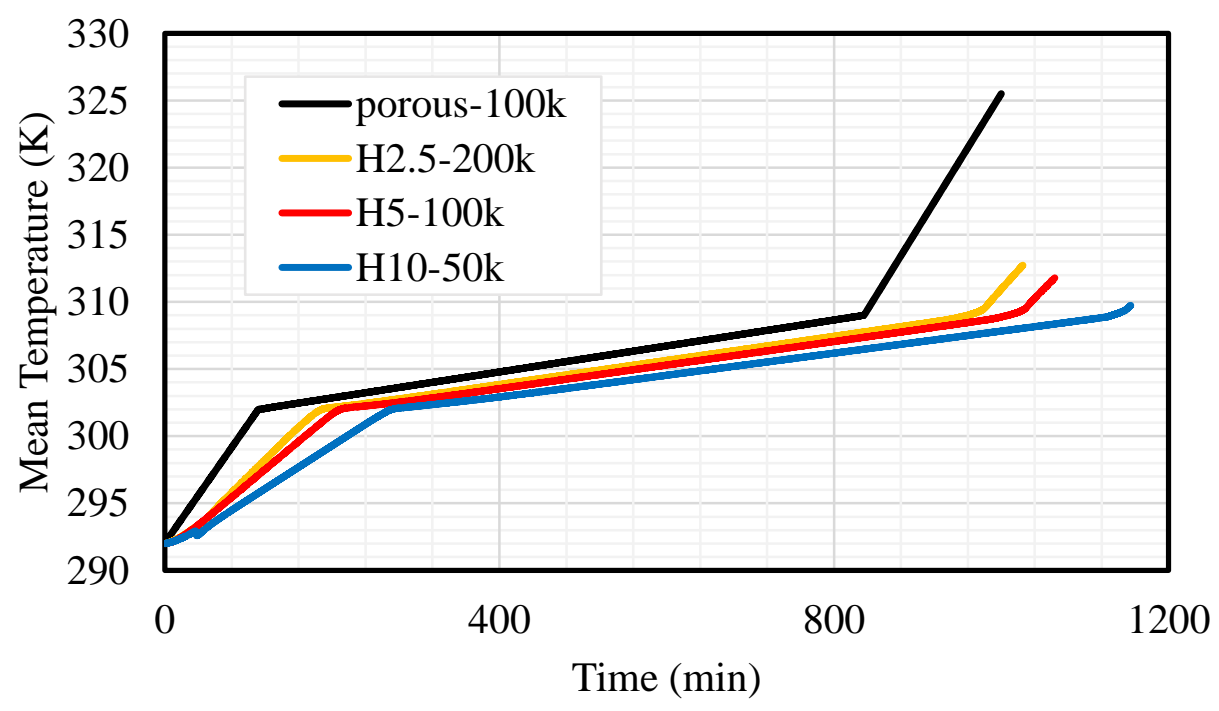

a) 


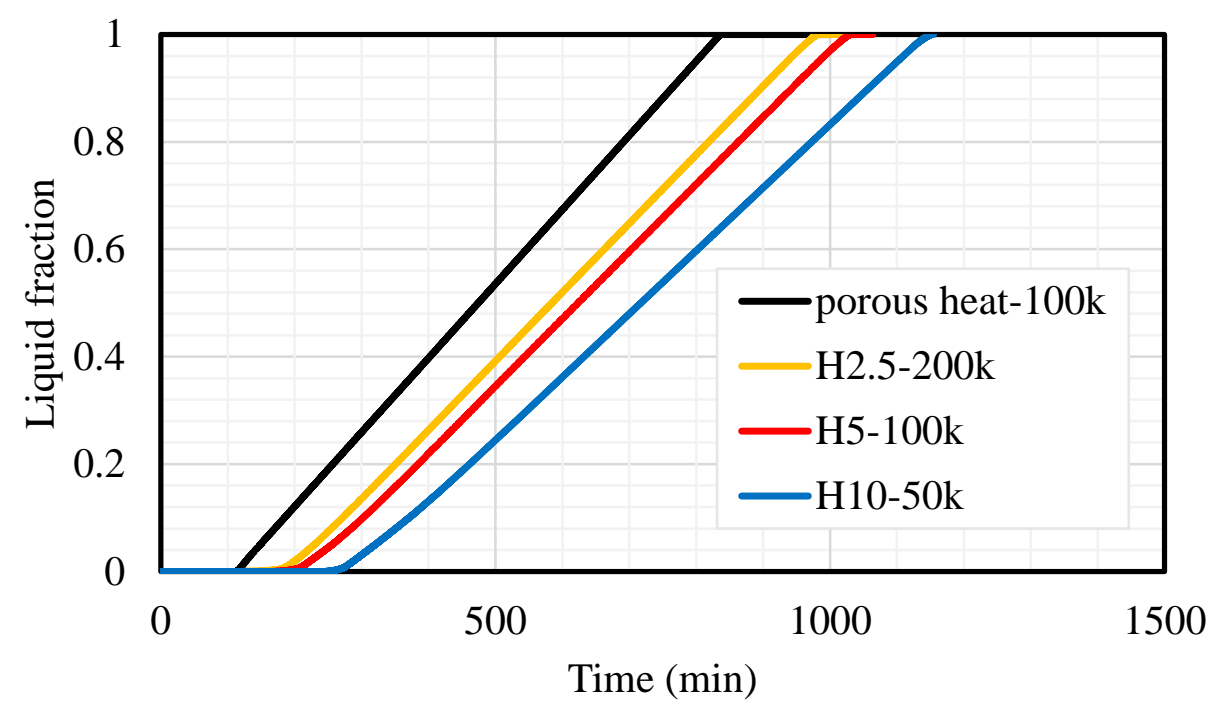

b)

Fig. 10. The variation of a) mean temperature and b) liquid fraction in terms of time for the system with a central heating element related to the heat generation rate with different sizes and heat generation rates with $\varepsilon=0.95 \%, \omega=30$ PPI

\section{Conclusion}

Effects of uniform internal heat generation based on Joule heating to porous foams embedded in PCM-based heat storage system were numerically investigated. The melting time was compared with the case of a localised central heating element considering the same PCM volume and the same heat generation rate. The results show an almost $21 \%$ reduction in the total melting time with the application of internal heat generation rate from the porous medium compared with the case of a central heating element for heat generation rate of $100 \mathrm{~kW} / \mathrm{m}^{3}$. Meanwhile, the melting time is not affected significantly by increasing the pore density of the metal foam. It is also observed that doubling the rate of heat generation could reduce the melting time up to $50 \%$ due to the uniformity of temperature distribution with the application of internal heat generation. For the analysis of central heating element with the same heat generation rate, the results reveal a superior effect of the heat generation rate than the heating 444 element dimensions on the melting time. For an identical volumetric heat source power of the 
445 localised heater, the rate of heat generation per volume is more effective compared with the 446 heating element size due to the presence of the porous medium. Results from the study would 447 serve as guidelines for the application of internal heat generation in broad PCM-based 448 applications including thermal control of electronic components, freezing of biological 449 tissues, and solar thermal energy storage systems. Furthermore, the study on the dimensions of 450 the central heating element provides effective approaches for a more efficient energy storage 451 system.

452

\section{References}

454 [1] Cornell B. Energy and Investing: Financing the Transition to Renewable Energy. 2019.

455 [2] Lyu P, Liu X, Qu J, Zhao J, Huo Y, Qu Z, et al. Recent advances of thermal safety of lithium 456 ion battery for energy storage. Energy Storage Materials. 2020.

457 [3] Sardari PT, Babaei-Mahani R, Giddings D, Yasseri S, Ardekani MM, Bahai H. Energy 458 recovery from domestic radiators using a compact composite metal Foam/PCM latent heat 459 storage. Journal of Cleaner Production. 2020:120504.

460 [4] Wang P, Wang X, Huang Y, Li C, Peng Z, Ding Y. Thermal energy charging behaviour of 461 a heat exchange device with a zigzag plate configuration containing multi-phase-change462 materials (m-PCMs). Applied energy. 2015;142:328-36.

463 [5] Zhang P, Xiao X, Meng ZN, Li M. Heat transfer characteristics of a molten-salt thermal 464 energy storage unit with and without heat transfer enhancement. Applied Energy. $465 \quad 2015 ; 137: 758-72$.

466 [6] Dincer I, Rosen M. Thermal energy storage: systems and applications: John Wiley \& Sons; 4672011. 
[7] Sharma A, Tyagi VV, Chen CR, Buddhi D. Review on thermal energy storage with phase

469

470

471

472

473

474

475

476

477

478

479

480

481

482

483

484

485

486

487

488

489

490

491

change materials and applications. Renewable and Sustainable Energy Reviews. 2009;13:31845.

[8] Tian Y, Zhao CY. A numerical investigation of heat transfer in phase change materials (PCMs) embedded in porous metals. Energy. 2011;36:5539-46.

[9] Mahdi JM, Nsofor EC. Multiple-segment metal foam application in the shell-and-tube PCM thermal energy storage system. Journal of Energy Storage. 2018;20:529-41.

[10] Mahdi JM, Nsofor EC. Melting of PCM with Nanoparticles in a Triplex-Tube Thermal Energy Storage System. Ashrae Transactions. 2016;122:215-24.

[11] El-Sebaii AA, Al-Ghamdi AA, Al-Hazmi FS, Faidah AS. Thermal performance of a single basin solar still with PCM as a storage medium. Applied Energy. 2009;86:1187-95.

[12] Ali HM, Ashraf MJ, Giovannelli A, Irfan M, Irshad TB, Hamid HM, et al. Thermal management of electronics: An experimental analysis of triangular, rectangular and circular pin-fin heat sinks for various PCMs. International Journal of Heat and Mass Transfer. 2018;123:272-84.

[13] Arshad A, Ali HM, Khushnood S, Jabbal M. Experimental investigation of PCM based round pin-fin heat sinks for thermal management of electronics: Effect of pin-fin diameter. International Journal of Heat and Mass Transfer. 2018;117:861-72.

[14] Warzoha RJ, Weigand RM, Fleischer AS. Temperature-dependent thermal properties of a paraffin phase change material embedded with herringbone style graphite nanofibers. Applied Energy. 2015;137:716-25.

[15] Shaibani AR, Keshtkar MM, Sardari PT. Thermo-economic analysis of a cold storage system in full and partial modes with two different scenarios: A case study. Journal of Energy Storage. 2019;24:100783. 

PCMs in a cavity subject to a non-uniform magnetic field using a moving grid technique.

494

495

496

497

498

499

500

501

502

503

504

505

506

507

508

509

510

511

512

513

514

515

516
Applied Mathematical Modelling. 2020;77:1936-53.

[17] Mehryan S, Tahmasebi A, Izadi M, Ghalambaz M. Melting behavior of phase change materials in the presence of a non-uniform magnetic-field due to two variable magnetic sources. International Journal of Heat and Mass Transfer. 2020;149:119184.

[18] Shahsavar A, Goodarzi A, Mohammed HI, Shirneshan A, Talebizadehsardari P. Thermal performance evaluation of non-uniform fin array in a finned double-pipe latent heat storage system. Energy. 2020;193:116800.

[19] Fang Y, Qu Z, Zhang J, Xu H, Qi G. Simultaneous charging and discharging performance for a latent thermal energy storage system with a microencapsulated phase change material. Applied Energy. 2020;275:115353.

[20] Sadeghi HM, Babayan M, Chamkha A. Investigation of using multi-layer PCMs in the tubular heat exchanger with periodic heat transfer boundary condition. International Journal of Heat and Mass Transfer. 2020;147:118970.

[21] Ghalambaz M, Chamkha AJ, Wen D. Natural convective flow and heat transfer of nanoencapsulated phase change materials (NEPCMs) in a cavity. International Journal of Heat and Mass Transfer. 2019;138:738-49.

[22] Chamkha A, Doostanidezfuli A, Izadpanahi E, Ghalambaz M. Phase-change heat transfer of single/hybrid nanoparticles-enhanced phase-change materials over a heated horizontal cylinder confined in a square cavity. Advanced Powder Technology. 2017;28:385-97.

[23] Talebizadeh Sardari P, Walker GS, Gillott M, Grant D, Giddings D. Numerical modelling of phase change material melting process embedded in porous media: Effect of heat storage size. Proceedings of the Institution of Mechanical Engineers, Part A: Journal of Power and Energy. 2019:0957650919862974. 

enhancement with multiple PCMs, cascaded metal foam and nanoparticles in the shell-andtube energy storage system. Applied Energy. 2020;257:113993.

Characteristics of a Double-Pipe Latent Heat Storage System with Sinusoidal Wavy Channels

522 Embedded in a Porous Medium. Energy. 2019;171:751-69.

[26] Ghalambaz M, Zhang J. Conjugate solid-liquid phase change heat transfer in heatsink

[27] Khudair WS, Al-Khafajy DGS. Influence of heat transfer on Magneto hydrodynamics oscillatory flow for Williamson fluid through a porous medium. Iraqi Journal of Science. 2018:389-97.

[28] Zhu F, Zhang C, Gong X. Numerical analysis on the energy storage efficiency of phase change material embedded in finned metal foam with graded porosity. Applied Thermal Engineering. 2017; 123:256-65.

532 [29] Zhang P, Meng ZN, Zhu H, Wang YL, Peng SP. Melting heat transfer characteristics of a composite phase change material fabricated by paraffin and metal foam. Applied Energy. 2017;185:1971-83.

535 [30] Zhao W, France DM, Yu W, Kim T, Singh D. Phase change material with graphite foam 536 for applications in high-temperature latent heat storage systems of concentrated solar power 537 plants. Renewable Energy. 2014;69:134-46. Change in Metal Foams. Journal of Heat Transfer. 2004;127:995-1004.

540 [32] Sharma SD, Sagara K. Latent heat storage materials and systems: a review. International 541 Journal of Green Energy. 2005;2:1-56. 
542 [33] Xu H, Zhang C, Wang N, Qu Z, Zhang S. Experimental study on the performance of a

543 solar photovoltaic/thermal system combined with phase change material. Solar Energy. $544 \quad 2020 ; 198: 202-11$.

545 [34] Xu H, Wang N, Zhang C, Qu Z, Cao M. Optimization on the melting performance of 546 triplex-layer PCMs in a horizontal finned shell and tube thermal energy storage unit. Applied 547 Thermal Engineering. 2020:115409.

548 [35] Wang X, Li B, Qu Z, Zhang J, Jin Z. Effects of graphite microstructure evolution on the 549 anisotropic thermal conductivity of expanded graphite/paraffin phase change materials and 550 their thermal energy storage performance. International Journal of Heat and Mass Transfer. $5512020 ; 155: 119853$.

[36] Moreno P, Castell A, Solé C, Zsembinszki G, Cabeza LF. PCM thermal energy storage 553 tanks in heat pump system for space cooling. Energy and Buildings. 2014;82:399-405.

554

[37] Sardari PT, Grant D, Giddings D, Walker GS, Gillott M. Composite metal foam/PCM energy store design for dwelling space air heating. Energy Conversion and Management. 2019;201:112151.

557

[38] Sardari PT, Giddings D, Grant D, Gillott M, Walker GS. Discharge of a composite metal 558 foam/phase change material to air heat exchanger for a domestic thermal storage unit. Renewable Energy. 2020;148:987-1001.

560 [39] Mettawee E-BS, Assassa GMR. Experimental study of a compact PCM solar collector. 561 Energy. 2006;31:2958-68.

562 [40] Jiji LM, Gaye S. Analysis of solidification and melting of PCM with energy generation. 563 Applied Thermal Engineering. 2006;26:568-75.

564 [41] Kalaiselvam S, Veerappan M, Arul Aaron A, Iniyan S. Experimental and analytical 565 investigation of solidification and melting characteristics of PCMs inside cylindrical 566 encapsulation. International Journal of Thermal Sciences. 2008;47:858-74. 

experimental investigation of melting with internal heat generation within cylindrical

569

570

571

572

573

574

575

576

577

578

579

580

581

582

583

584

585

586

587

588

589

590

591 enclosures. Applied Thermal Engineering. 2014;67:587-96.

[43] Bechiri M, Mansouri K. Analytical study of heat generation effects on melting and solidification of nano-enhanced PCM inside a horizontal cylindrical enclosure. Applied Thermal Engineering. 2016;104:779-90.

[44] Shahsavar A, Al-Rashed AAAA, Entezari S, Sardari PT. Melting and Solidification Characteristics of a Double-Pipe Latent Heat Storage System with Sinusoidal Wavy Channels Embedded in a Porous Medium. Energy. 2019.

[45] Py X, Olives R, Mauran S. Paraffin/porous-graphite-matrix composite as a high and constant power thermal storage material. International Journal of Heat and Mass Transfer. 2001;44:2727-37.

[46] Talebizadeh Sardari P, Walker G, Gillott M, Grant D, Giddings D. Numerical modelling of phase change material melting process embedded in porous media: Effect of heat storage size. Proc IMechE Part A: J Power and Energy. 2019:1-19.

[47] Shahsavar A, Al-Rashed AAAA, Entezari S, Sardari PT. Melting and solidification characteristics of a double-pipe latent heat storage system with sinusoidal wavy channels embedded in a porous medium. Energy. 2019;171:751-69.

[48] Mahdi JM, Nsofor EC. Melting enhancement in triplex-tube latent heat energy storage system using nanoparticles-metal foam combination. Applied Energy. 2017;191:22-34.

[49] Mat S, Al-Abidi AA, Sopian K, Sulaiman MY, Mohammad AT. Enhance heat transfer for PCM melting in triplex tube with internal-external fins. Energy Conversion and Management. 2013;74:223-36.

[50] Ye W-B, Zhu D-S, Wang N. Numerical simulation on phase-change thermal storage/release in a plate-fin unit. Applied Thermal Engineering. 2011;31:3871-84. 
592

593

594

595

596

597

598

599

600

601

602

603

604

605

606

607

608

609

610

611

612

613

614

615

616

[51] Assis E, Katsman L, Ziskind G, Letan R. Numerical and experimental study of melting in a spherical shell. International Journal of Heat and Mass Transfer. 2007;50:1790-804.

[52] Al-Abidi AA, Mat S, Sopian K, Sulaiman MY, Mohammad AT. Internal and external fin heat transfer enhancement technique for latent heat thermal energy storage in triplex tube heat exchangers. Applied Thermal Engineering. 2013;53:147-56.

[53] Nield DA, Bejan A. Convection in Porous Media: Springer International Publishing; 2017. [54] Alsabery AI, Mohebbi R, Chamkha AJ, Hashim I. Effect of local thermal non-equilibrium model on natural convection in a nanofluid-filled wavy-walled porous cavity containing inner solid cylinder. Chemical Engineering Science. 2019;201:247-63.

[55] Zhao CY, Lu W, Tian Y. Heat transfer enhancement for thermal energy storage using metal foams embedded within phase change materials (PCMs). Solar Energy. 2010;84:140212.

[56] Liu Z, Yao Y, Wu H. Numerical modeling for solid-liquid phase change phenomena in porous media: Shell-and-tube type latent heat thermal energy storage. Applied Energy. $2013 ; 112: 1222-32$.

[57] Xu Y, Li M-J, Zheng Z-J, Xue X-D. Melting performance enhancement of phase change material by a limited amount of metal foam: Configurational optimization and economic assessment. Applied Energy. 2018;212:868-80.

[58] Liu Z, Ma C. Numerical analysis of melting with constant heat flux heating in a thermal energy storage system. Energy Conversion and Management. 2002;43:2521-38.

[59] Pal D, Joshi YK. Melting in a side heated tall enclosure by a uniformly dissipating heat source. International Journal of Heat and Mass Transfer. 2001;44:375-87.

[60] Shatikian V, Ziskind G, Letan R. Numerical investigation of a PCM-based heat sink with internal fins: Constant heat flux. International Journal of Heat and Mass Transfer. 2008;51:1488-93. 
617 [61] Li WQ, Qu ZG, He YL, Tao WQ. Experimental and numerical studies on melting phase 618 change heat transfer in open-cell metallic foams filled with paraffin. Applied Thermal 619 Engineering. 2012;37:1-9.

620

621 